\begin{tabular}{|c|c|c|c|c|c|c|}
\hline & Short bowel (\%) & Motility disorder (\%) & Re-transplant (\%) & Desmoids (\%) & Other $(\%)$ & Total \\
\hline 2006 & $1(100)$ & & & & & 1 \\
\hline 2007 & & $1(50)$ & & & $1(50)$ & 2 \\
\hline 2008 & $1(30)$ & & & $2(70)$ & & 3 \\
\hline 2009 & $3(60)$ & & $1(20)$ & & $1(20)$ & 5 \\
\hline 2010 & $5(83)$ & & 1 (17) & & & 6 \\
\hline 2011 & $6(75)$ & & & & $2(25)$ & 8 \\
\hline 2012 & $2(33)$ & & 1 (17) & 1 (17) & $2(33)$ & 6 \\
\hline 2013 & $8(50)$ & $3(19)$ & 1 (6) & 1 (6) & $3(19)$ & 16 \\
\hline
\end{tabular}

Introduction Small bowel transplantation (SBT) was first performed in the UK in Cambridge in 1991. Recipients mow undergo small bowel (SBT), liver and small bowel (LSBT), modified multivisceral (MMVT - small bowel, stomach, pancreas, no liver) and multivisceral (MVT - intestine, stomach, pancreas and liver) transplantation. Cambridge is the only UK centre offering MVT in adults.

The main indications for referral to a transplant centre are:

1. Irreversible intestinal failure plus life threatening complications of parenteral nutrition (PN).

2. Extensive surgery requiring partial or complete evisceration.

Methods Prospective data was collected from all patients who underwent intestinal and multivisceral transplantation at Addenbrooke's Hospital between 2003 and 2013. All patients are discussed and indications for transplantation agreed prior to listing at NASIT (National Adult Small Intestinal Transplant forum).

Results 47 transplants were performed on 43 patients; 4 procedures $(9 \%)$ were re-transplantation for a primary non-functioning graft $(2 / 4)$ or acute rejection (2/4). The indications for transplant are presented below:

Sixteen transplants were performed in 2013 - MVT (57\%), SBT (31\%), LSB (6\%) and MMVT (6\%). 50\% of these were due to short bowel - arterial ischaemia (50\%), Crohn's (26\%), venous ischaemia (12\%) and other short bowel (12\%). Colon is now routinely included in the graft to aid fluid balance and does not preclude endoscopic surveillance for rejection.

Conclusion The number of small bowel and multivisceral transplants performed over the last 10 years has increased, and more than doubled in 2013. Short bowel remains the commonest indication for transplantation. Historically this was mainly due to Crohn's disease however in 2013, it was mainly due to ischaemia; this trend was reflected worldwide. In our cohort, an increase in acute arterial thromboses causing coel$\mathrm{iac} /$ mesenteric ischaemia resulted in 3 recipients being listed urgently for MVT. There has also been an increase in the number of patients referred with portal vein thromboses extending into the superior mesenteric vein, precluding liver transplant alone.

Disclosure of Interest None Declared.

\section{PTH-125 SURVIVAL FOLLOWING INTESTINAL AND MULTIVISCERAL TRANSPLANTATION AT ADDENBROOKE'S HOSPITAL, CAMBRIDGE, UK}

${ }^{1} \mathrm{C}$ Rutter*, 'L Sharkey, ${ }^{2} \mathrm{~A}$ Butler, ${ }^{2} \mathrm{~N}$ Russell, ${ }^{1} \mathrm{C}$ Pither, ${ }^{1} \mathrm{~J}$ Green, ${ }^{1} \mathrm{~S}$ Duncan, ${ }^{1} \mathrm{D}$ Bond, ${ }^{1} \mathrm{~B}$ Chukualim, '1J Woodward, ${ }^{3} \mathrm{~S}$ Gabe, ${ }^{2} \mathrm{~N}$ Jamieson, ${ }^{1} \mathrm{~S}$ Middleton. 'Gastroenterology, Addenbrooke's Hospital, Cambridge, UK; ${ }^{2}$ Transplant Surgery, Addenbrooke's Hospital, Cambridge, UK; ${ }^{3}$ Lennard-Jones Intestinal Failure Unit, St Mark's Hospital, London, UK

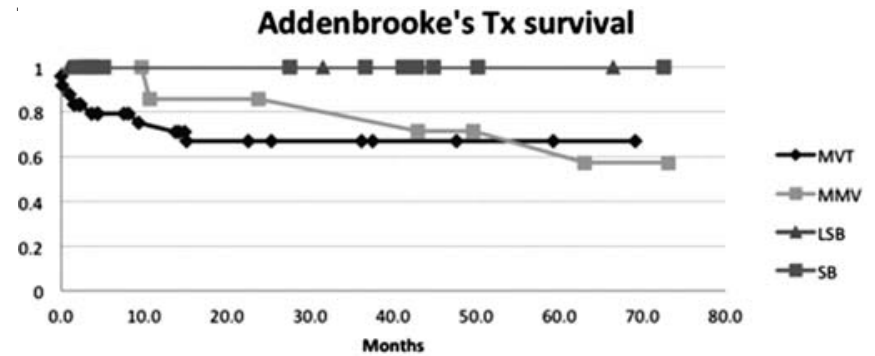

Abstract PTH-125 Figure 1

Introduction Small intestinal transplantation (SBT) was first undertaken in the UK in Cambridge in 1991. Since the introduction of new immunosuppressive agents around the millenium, results have improved and we present our experience over the last 10 years. Since 2003, 47 tranplants have been performed on 43 patients. Grafts include small bowel or small bowel/colon (SBT), liver and small bowel (LSBT), modified multivisceral (MMVT - small bowel, stomach, pancreas, no liver) and multivisceral (MVT - intestine, stomach, pancreas and liver) transplantation. Cambridge is the only UK centre offering MVT in adults.

Methods A review of all patients who underwent small intestine and multivsiceral transplantation at Addenbrooke's Hospital between 2003 and 2013. Kaplan-Meier survival data are shown for each group of organs transplanted.

Results Five year survival for all patients transplanted is $77 \%$. Survival curves for each organ group transplanted is graphed below:

Conclusion Five year survival in our patients transplanted since 2003 is 100\% for SBT and LSBT and 65\% for MVT, compared with international registry survival figures of 59\% (SBT and LSBT combined) and $22 \%$ respectively.

In recent years we have also experienced an increase in the number of urgent transplants performed and these patients are often critically unwell at the time of surgery. Our centre undertakes a relatively large number of procedures and this, coupled with a particular focus on multidisciplinary team working, may account in part for our favourable survival figures.

Disclosure of Interest None Declared.

\section{PTH-126 ASSESSMENT OF CARDIOVASCULAR RISK OF PATIENTS ON HOME PARENTERAL NUTRITION}

${ }^{1}$ DS Chilkunda*, ${ }^{2}$ S Scantlebury, ${ }^{2} \mathrm{~J}$ Wood, ${ }^{2} \mathrm{R}$ Reid, ${ }^{2} \mathrm{H}$ Lynch, ${ }^{2} \mathrm{G}$ Sayell, ${ }^{2} \mathrm{G}$ Ani, ${ }^{3} \mathrm{D}$ Burke, ${ }^{4} \mathrm{C}$ Donnellan. ${ }^{1}$ Gastroenterology, St James's University Hospital NHS Trust, Leeds, UK; ${ }^{2}$ Nutrition Team, St. James's University Hospital NHS Trust, Leeds, UK; ${ }^{3}$ Colorectal Surgery and Nutrition, St. James's University Hospital NHS Trust, Leeds, UK; ${ }^{4}$ Gastroenterology and Nutrition, St. James's University Hospital NHS Trust, Leeds, UK 